\section{Kompass}

Nutrition \& Dietetics
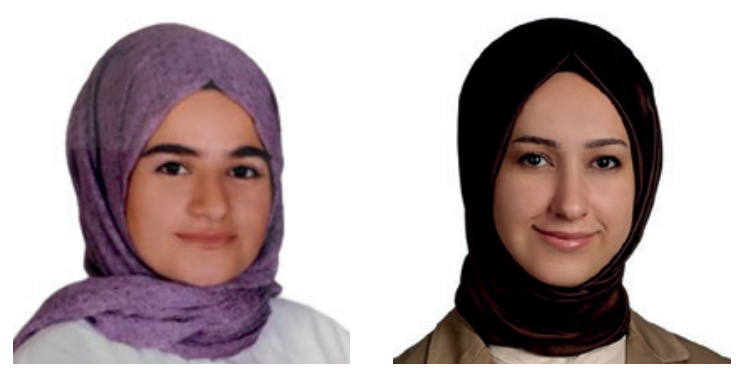

\title{
Is Digital Health Intervention Effective in Inflammatory Bowel Disease Patients?
}

\author{
Bilge Roj Gunerhanal $^{\mathrm{a}}$ Merve Guney-Coskun ${ }^{\mathrm{b}}$
}

a Neubrandenburg Institute of Evidence-based Dietetics (NIED), University of Applied Sciences Neubrandenburg, Neubrandenburg, Germany; ${ }^{b}$ Faculty of Health Science, Department of Nutrition \& Dietetics, Istanbul Medipol University, Istanbul, Turkey

Abstract from Zhen J, Marshall JK, Nguyen GC, et al.: Impact of digital health monitoring in the management of inflammatory bowel disease. J Med Syst. 2021;45(2):23.

\section{Keywords \\ Digital health monitoring · eHealth · HealthPROMISE · IBD · \\ Inflammatory bowel disease $\cdot$ Telemedicine $\cdot$ eHealth}

\begin{abstract}
Technological advances now permit self-management strategies using mobile applications which could greatly benefit patient care. The purpose of this study was to investigate whether the use of the inflammatory bowel disease (IBD) digital health monitoring platform, HealthPROMISE, leads to better quality of care and improved health outcomes in IBD patients. IBD patients were recruited in gastroenterology clinics and asked to install the HealthPROMISE application onto their smartphones. Patient satisfaction, quality of care, quality of life, patient symptoms, and resource utilization metrics were collected throughout the study and sent directly to their healthcare teams. Patients with abnormal symptom/SIBDQ scores were flagged for their physicians to follow up.
\end{abstract}

After one-year, patient outcome metrics were compared to baseline values. Overall, out of 59 patients enrolled in the study, 32 patients (54\%) logged into the application at least once during the study period. The number of IBD-related ER visits/hospitalizations in the year of use compared to the prior year demonstrated a significant decrease from $25 \%$ of patients (8/32) to 3\% (1/32) ( $p$ $=0.03$ ). Patients also reported an increase in their understanding of the nature/causes of their condition after using the application $(p=0.026)$. No significant changes were observed in the number of quality indicators met ( $p=0.67)$ or in SIBDQ scores ( $p=0.48$ ). Given the significant burden of IBD, there is a need to develop effective management strategies. This study demonstrated that digital health monitoring platforms may aid in reducing the number of ER visits and hospitalizations in IBD patients.

(c) 2021 The Author(s), under exclusive license to Springer Science+Business Media, LLC part of Springer Nature 


\section{Knowledge Transfer}

\section{Background}

Crohn's disease (CD) and ulcerative colitis (UC) are the two main forms of inflammatory bowel disease (IBD) which is a chronic intestinal disorder with remission and relapse periods [1]. IBD is associated with symptoms such as diarrhea, abdominal pain, weight loss, bloody stools, and various extraintestinal manifestations. IBD is associated with mood disorders, emotional distress, as well as lower quality of life. To prevent relapses and symptoms, current treatment focuses on 'treat-to-target' strategies before it is too late. With the development of technology in the field of health and the spread of treatments on the digital platform due to COVID-19, interventions related to effective self-management strategies for outpatient care have become more important. This study aimed to investigate the efficacy of HealthPROMISE - an IBD health monitoring platform for use in clinical practice and to evaluate whether its use leads to better quality of care and improved health outcomes [2].

\section{HealthPROMISE System}

HealthPROMISE was developed as a cloud-based digital therapeutics and IBD monitoring application that can be downloaded onto phones. Patients can track their symptoms, medication, doctor's visits, emergency room visits, and hospitalizations. Additionally, the app allows calculation of quality of life scores (based on the Short Inflammatory Bowel Disease Questionnaire (SIBDQ)) and quality of care scores (based on IBD quality indicators developed by the Canadian Association of Gastroenterology). Furthermore, through this application, physicians are able to follow up their patients and give them feedback.

\section{Potentials and Limitations of Digital Health Interventions for IBD}

IBD patients need frequent medical care. eHealth technologies represent a promising new tool for efficient and reliable treatment and follow-up. In the course of the COVID-19 pandemic, eHealth tools have become an essential part of healthcare, and patients now tend to use these tools.

One limitation of the current study assessing digital health monitoring systems was that only people with comfortable access to technology and the internet took part. The demographic characteristics (rural-urban) of the participants were not considered in the calculations. A more diverse and wider sample size should be selected for future studies. Cost factors should also be taken into account, and studies should be developed accordingly [3].

\section{Future Perspectives of Dietetic Practice}

Digital health has existed in the form of medical informatics and simple telehealth for over two decades; however, a proliferation of literature on this topic has only been seen in the last 5 years [4]. Some innovations showcase the potential for improving diet practices through digital health. For example, since 2017, dietitians in the UK have had access to free, on-demand webinars for patients (www.patientwebinars.co.uk) as part of their treatment and/or education regarding certain gastroenterological conditions [5]. On the other hand, a 2019 survey by Abrahams et al. [6] demonstrated that most dietitians did not consider technology to play an important role in their current practice. The most important reasons for this were fear of the profession losing value and lack of financial standards while the unsystematic approach may not have a lasting effect on patients.

The COVID-19 pandemic has provided the opportunity for healthcare's digital revolution with the sudden accelerated expansion of telehealth, telemedicine, and other digital health tools. A survey conducted in 2015 showed that 58\% of smartphone users downloaded a health-related app to manage their lifestyle [7]. The systematic development and active application of the digital platform, which was also emphasized in the pandemic, will reach wider masses in a short time and with maximum efficiency.

\section{Disclosure Statement}

The authors hereby declare that there are no conflicts of interest with regard to this commentary.

\section{References}

1 Guan Q: A Comprehensive review and update on the pathogenesis of inflammatory bowel disease. J Immunol Res. 2019;2019:7247238.

2 Zhen J, Marshall JK, Nguyen GC, et al.: Impact of digital health monitoring in the management of inflammatory bowel disease. J Med Syst. 2021;45(2):23.

3 Banbury A, Roots A, Nancarrow S: Rapid review of applications of e-health and remote monitoring for rural residents. Aust J Rural Health. 2014;22(5):211-222.

4 Ahmadvand A, Kavanagh D, Clark M, et al.: Trends and visibility of "digital health" as a keyword in articles by JMIR publications in the new millennium: bibliographic-bibliometric analysis. J Med Internet Res. 2019;21(12):e10477.

5 Williams M, Barclay Y, Harper L, et al.: Feasibility, acceptability and cost efficiency of using webinars to deliver first-line patient education for people with Irritable Bowel Syndrome as part of a dietetic-led gastroenterology service in primary care. J Hum Nutr Diet. 2020;33(6):758-766.

6 Abrahams M, Frewer LJ, Bryant E, et al.: Personalised Nutrition Technologies and Innovations: A Cross-National Survey of Registered Dietitians. Public Health Genomics. 2019;22(3-4):119-131.

7 Krebs P, Duncan DT: Health App Use Among US Mobile Phone Owners: A National Survey. JMIR Mhealth Uhealth. 2015;3(4):e101.

\section{Correspondence to:}

Bilge Roj Gunerhanal, bilgegunerhanal @ gmail.com

Merve Guney-Coskun, merve.guney @ medipol.edu.tr 